\title{
African swine fever virus p10 protein exhibits nuclear import capacity and accumulates in the nucleus during viral infection
}

\author{
Isabel Nunes-Correia $^{\mathrm{a}, \mathrm{b}}$, Javier M. Rodríguez ${ }^{\mathrm{c}}$, Ana Eulálio ${ }^{\mathrm{a}, \mathrm{b}}$, Ana L. Carvalho ${ }^{\mathrm{a}, \mathrm{d}}$, \\ Vitaly Citovsky ${ }^{\mathrm{e}}$, Sérgio Simões ${ }^{\mathrm{a}, \mathrm{f}}$, Carlos Faro ${ }^{\mathrm{a}, \mathrm{b}}$, María L. Salas ${ }^{\mathrm{c}}$, \\ Maria C. Pedroso de Lima ${ }^{a, b, *}$ \\ ${ }^{\text {a } C e n t r o ~ d e ~ N e u r o c i e ̂ n c i a s ~ e ~ B i o l o g i a ~ C e l u l a r, ~ U n i v e r s i d a d e ~ d e ~ C o i m b r a, ~ 3004-517 ~ C o i m b r a, ~ P o r t u g a l ~}$ \\ ${ }^{\mathrm{b}}$ Departamento de Bioquímica, Universidade de Coimbra, 3001-401 Coimbra, Portugal

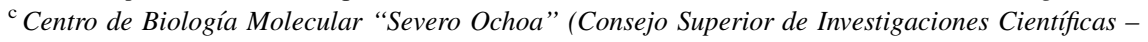 \\ Universidad Autónoma de Madrid), Universidad Autónoma, Cantoblanco, 28049 Madrid, Spain \\ ${ }^{\mathrm{d}}$ Departmento de Zoologia, Universidade de Coimbra, 3004-504 Coimbra, Portugal \\ ${ }^{\mathrm{e}}$ Department of Biochemistry and Cell Biology, State University of New York, Stony Brook, NY 11794-5215, USA \\ ${ }^{\mathrm{f}}$ Departmento de Tecnologia Farmacêutica, Faculdade de Farmácia, \\ Universidade de Coimbra, 3000-295 Coimbra, Portugal
}

Received 22 October 2007; received in revised form 7 December 2007; accepted 19 December 2007

\begin{abstract}
African swine fever virus (ASFV), a large enveloped DNA-containing virus, infects domestic and wild pigs, and multiplies in soft ticks, causing an economically relevant hemorrhagic disease. Evaluation of the nuclear import ability of ASFV p10 protein was the major purpose of the present work. Two approaches were used to determine if p10 protein is imported into the nucleus by an active process: a yeast-based nuclear import assay and the determination of the subcellular localization of p10 protein in mammalian cells by fluorescence microscopy. The results obtained clearly demonstrate that p10 protein is actively imported into the nucleus, both in yeast and mammalian cells. Experiments aiming at identifying the critical residues responsible for the nuclear import of ASFV p10 protein indicate that the amino acids comprised between the positions 71 and 77 are important, although not sufficient, for the protein active nuclear import. In ASFV-infected cells, the p10 protein strongly accumulates in the nucleus at late times post-infection, indicating that p10 protein may accomplish an important function inside the nucleus during the late phase of the viral replication cycle.
\end{abstract}

(C) 2008 Elsevier B.V. All rights reserved.

Keywords: African swine fever virus; Nuclear import; p10 protein

* Corresponding author at: Departmento de Bioquímica, Universidade de Coimbra, Apartado 3126, 3001-401 Coimbra, Portugal. Tel.: +351 239820190; fax: +351239853607.

E-mail address: mdelima@ci.uc.pt (M.C. Pedroso de Lima). 


\section{Introduction}

African swine fever virus (ASFV), the only member of the family Asfarviridae has been described as a missing evolutionary link between the families Poxviridae and Iridoviridae, since ASFV shares the genomic organization and the striking icosahedral symmetry of those families, respectively (Salas et al., 1999). The early descriptions of ASFV infections of domestic pigs were of an acute hemorrhagic fever, which caused approximately $100 \%$ mortality. In the last years, an increased number of isolates were identified, where the virus pathogenesis may range from rapidly fatal to subclinical, chronic or no explicit symptoms of disease (Leitão et al., 2001; Dixon et al., 2004).

The virus particle possesses a complex structure composed of several concentric domains with an overall icosahedral shape and an average diameter of 200 nm (Carrascosa et al., 1984; Andrés et al., 1997, 1998). The viral core is composed of a DNAcontaining nucleoid surrounded by a thick protein layer, the core shell, and is wrapped by a lipid envelope and an icosahedral capsid (Carrascosa et al., 1984; Andrés et al., 1997). Extracellular ASFV particles usually possess an additional membrane acquired by budding through the host cell plasma membrane (Breese and Pan, 1978). The viral genome is a single molecule of double-stranded DNA ranging in size from 170 to $190 \mathrm{kbp}$ with terminal inverted repetitions and terminal cross-links, which encodes approximately 50 structural proteins (Yáñez et al., 1995). Some of the ASFV proteins have been shown to exhibit DNA-binding activity like the structural p10 protein which is localized in the viral nucleoid (Muñoz et al., 1993; Andrés et al., 2002).

Although ASFV has been considered for many years as a virus that replicates exclusively in the cytoplasm of infected cells, the observation that the virus does not replicate in enucleated Vero cells nor is its DNA synthesized (Ortin and Viñuela, 1977) raised the possibility that the nucleus is involved in viral DNA replication. More recently, it has been reported that the replication of viral DNA is initiated in the nucleus, a stage which is followed by a longer cytoplasmic phase (García-Beato et al., 1992; Rojo et al., 1999). The mechanisms of ASFV viral genome nuclear import and export are still unknown. However, as described for other viral genomes, this nucleuscytoplasm transport is most likely mediated by viral proteins associated with the ASFV DNA (Whittaker and Helenius, 1998).

The active and bidirectional transport of proteins across the nuclear pore complex (NPC) is a tightly regulated process that plays a vital role in eukaryotic cells. Small molecules like metabolites, ions and small proteins can diffuse through the 9-nm NPC channel in either direction, between the nucleus and cytoplasm, which sets an upper limit for free diffusion to $\sim 45-$ $60 \mathrm{kDa}$. However, particles as large as $36 \mathrm{~nm}$ in diameter can be transported through the NPC by a selective and energy-dependent mechanism that acts to translocate macromolecules into and out of the nucleus. The active nucleus-cytoplasm transport is mediated by shuttling receptors that interact with localization signals on cargo molecules, RanGTP and proteins of the NPC (Görlich and Kutay, 1999; Macara, 2001).

The active import of proteins across the nuclear envelope requires the presence of specific targeting sequences within the protein, named nuclear localization signals (NLSs), even when the proteins are small enough to diffuse through the NPC (Görlich et al., 1996). There are at least three types of NLSs, which are characteristically rich in the basic amino acids lysine and arginine (Dingwall and Laskey, 1991). The first type, referred as classical NLS, is composed of a basic amino acid stretch $(\mathrm{K} / \mathrm{R})_{4-6}$ preceded by a glycine, proline or an acidic amino acid residue, similar to the NLS of simian virus 40 (SV40) large T antigen (Kalderón et al., 1984). The second type is a bipartite sequence composed of two basic amino acid stretches separated by an intervening nonconserved 10-12 amino acid spacer: $(\mathrm{K} / \mathrm{R})_{2} \mathrm{X}_{10-12}(\mathrm{~K} / \mathrm{R})_{3}$, an example of this being the Xenopus Laevis nucleoplasmin (Dingwall et al., 1988). The third type is a less well-conserved sequence with few basic residues such as that of the adenovirus E1A protein (KRPRP) (Lyons et al., 1987). However, many other NLSs have been identified that differ from these sequences with respect to size and/or the content in basic amino acids (Makkerh et al., 1996; Michael et al., 1997). In addition to linear NLSs, discontinuous epitopes that come together upon folding into tertiary structure have been described to contribute to the nuclear import of histone proteins (Baake et al., 2001). Therefore, it is 
difficult to identify an NLS without experimental evidence.

ASFV p10 structural protein is encoded by the K78R gene and its DNA binding capacity has been described for both double- and single-stranded DNA (Muñoz et al., 1993). This protein is an extremely hydrophilic polypeptide with a relatively high content of basic residues (23\%), namely lysine residues (Muñoz et al., 1993). Motivated by such characteristics, we decided to investigate whether ASFV p10 protein exhibits nuclear transport activity.

\section{Materials and methods}

\subsection{Plasmid constructs}

pNIA, pNIAE2, pNIA + E2, pNIAD2(ant) and pNIAGFP plasmids were described previously (Rhee et al., 2000; Eulálio et al., 2004).

pNIAGFPp10 and GFPp10 were generated by cloning p10 cDNA, downstream of the green fluorescent protein (GFP). The cDNA encoding p10 was amplified by PCR with ASFV Lisbon 60 strain DNA as a template and using specific primers. The primers introduced BamHI and PstI recognition sites, respectively, at the $5^{\prime}$ and $3^{\prime}$ ends of the amplified DNA sequence. The DNA fragment was then cloned into the BamHI/PstI restriction sites of the plasmid for nuclear import (pNIA) containing the GFP (pNIAGFP).

GFP $\beta$ GUS was generated by cloning $\beta$-glucuronidase protein ( $\beta$ GUS) cDNA downstream of GFP. The cDNA encoding $\beta$ GUS was amplified by PCR using specific primers that introduced BamHI and BgIII recognition sites, respectively, at the $5^{\prime}$ and $3^{\prime}$ ends of the amplified DNA sequence. The DNA fragment was then cloned into the BglII restriction site of pEGFP-C2 (Clontech).

ASFV p10 protein and the various fragments used for mapping the NLS of $\mathrm{p} 10$ protein were amplified by $\mathrm{PCR}$, using specific primers that introduced BamHI or BglII and PstI recognition sites, respectively, at the $5^{\prime}$ and $3^{\prime}$ ends of the amplified DNA sequence. The DNA fragments were then cloned into the BglII/PstI restriction sites of GFP $\beta$ GUS.

Proper framing and accuracy of the sequences of all DNA constructs were confirmed by DNA sequencing.
All primers used in this study and construction details are available on request.

Purification of plasmid DNA for sequencing and transfection experiments was performed using Plasmid Midi Kit (Qiagen) or Wizard ${ }^{\mathbb{R}}$ Plus SV Minipreps DNA Purification System (Promega).

\subsection{Yeast nuclear import assay}

The yeast nuclear import assay was performed as described previously (Rhee et al., 2000). Briefly, the pNIA-derived constructs were transformed into Saccharomyces cerevisiae strain L40, which contains the two LexA-inducible genes HIS3 and lacZ (Hollenberg et al., 1995), by the lithium acetate method (Kaiser et al., 1994). The transformed yeasts were then plated on selective medium without tryptophan. Since pNIA contain the TRPI gene, only transformed yeasts can grow on this medium.

After growth, a few colonies were spread on minimal medium without tryptophan and in parallel were plated on minimal medium lacking both tryptophan and histidine, and supplemented with $10 \mathrm{mM}$ 3-amino-1,2,4-triazole (3AT; Sigma), a repressor of yeast endogenous production. Yeast growth in the absence of histidine was evaluated.

Additionally, yeast cells plated on tryptophandeficient medium were transferred to nitrocellulose filters and assayed for $\beta$-galactosidase activity (Breeden and Nasmyth, 1985). Briefly, after disruption of yeast cell membrane by incubation at $-70{ }^{\circ} \mathrm{C}$ for $30 \mathrm{~min}$, the yeast lysate was incubated with $\beta$ galactosidase substrate 5-bromo-4-chloro-3-indolylD-galactopyranoside (X-GAL; Sigma), and blue color development was evaluated.

For quantitative determination of $\beta$-galactosidase activity, an enzymatic assay was performed in liquid cultures (Stachel et al., 1985). After growth in liquid medium without tryptophan, yeast cells were disrupted, and the $\beta$-galactosidase chromogenic substrate $o$-nitrophenyl-D-galactopyranoside (ONPG; Sigma) was added in excess. After incubation at $30{ }^{\circ} \mathrm{C}$, the reaction was stopped by raising the $\mathrm{pH}$ to 11 , which inactivates $\beta$-galactosidase activity. The $\beta$-galactosidase activity was calculated according to the following equation (Miller, 1972): $\beta$-galactosidase units $=1000 \times V \times \mathrm{OD}_{600} \times \mathrm{OD}_{420} / t$, where $V$ is the volume of the sample used in the assay (in $\mathrm{ml}$ ), $\mathrm{OD}_{600}$ 
is the optical density at $600 \mathrm{~nm}$ of the yeast cell culture at the start of the assay, $\mathrm{OD}_{420}$ is the optical density at $420 \mathrm{~nm}$ of the sample measured after the incubation of yeast cell lysate with ONPG and $t$ is the time of incubation (in min).

\subsection{Cell culture and viruses}

For transfection experiments, Vero cells (ATCC CCL81) were grown in VP-SFM (GIBCO) supplemented with L-Glutamine (GIBCO) and $1 \%$ fetal calf serum (FCS) or in Dulbecco's modified Eagle's medium (DMEM) (Sigma) supplemented with $10 \%$ fetal calf serum (FCS). For viral infection, Vero cells were grown in DMEM (GIBCO) supplemented with $10 \%$ FCS, which was reduced to $2 \%$ during viral infection. Highly purified extracellular ASFV BA71V strain, adapted to grow in Vero cells, was obtained by Percoll equilibrium centrifugation (Carrascosa et al., 1985) and used in the infections.

\subsection{Transfection}

Transfection experiments were performed using Lipofectamine $^{\mathrm{TM}} 2000$ (Invitrogen), according to manufacturer's instructions. Vero cells were incubated $36 \mathrm{~h}$ to allow gene expression.

\subsection{Fluorescence microscopy}

For fluorescence analysis of GFP $\beta$ GUS fusion proteins, Vero cells were washed with phosphatebuffered saline (PBS), fixed with $4 \%$ paraformaldehyde for $30 \mathrm{~min}$ at room temperature, and rinsed with PBS. Then, coverslips were mounted with Mowiol 4 88 (Fluka) on glass slides and the preparations were examined with a Zeiss Axioskop2 plus fluorescence microscope, at a $600 \times$ magnification. Images were processed using Adobe Photoshop software.

\subsection{Immunofluorescence microscopy}

Pre-confluent Vero cells grown on coverslips were infected with ASFV at 10 pfu per cell. At different times post-infection, the cells were fixed with $2 \%$ paraformaldehyde for $1 \mathrm{~h}$ at room temperature or with $2 \%$ paraformaldehyde, under the same conditions, and then with methanol at $-20{ }^{\circ} \mathrm{C}$ for $5 \mathrm{~min}$. In the case where no methanol was used, cells were permeabilized with PBS/0.2\% Triton X-100 for $5 \mathrm{~min}$ at room temperature. After being washed with PBS, the cells were blocked for 30 min with $1 \%$ cold fish skin gelatin (Sigma) and 10\% normal goat serum (NGS) (Jackson Immunoresearch) in PBS with $0.1 \%$ Triton X-100. Vero cells were then incubated for $1 \mathrm{~h}$ with rabbit polyclonal serum anti-p10 (Andrés et al., 2002) and mouse monoclonal antibody 17LD3 anti-p72 (Sanz et al., 1985) diluted $1 / 1000$ and $1 / 200$, respectively, in blocking solution without NGS. After extensive washing with $\mathrm{PBS} / 0.1 \%$ Triton $\mathrm{X}-100$, cells were blocked for 30 min with $1 \%$ cold fish skin gelatin and $10 \%$ of NGS in PBS with $0.1 \%$ Triton X-100 and then incubated for $1 \mathrm{~h}$ with Alexa 488 goat anti-rabbit immunoglobulin $\mathrm{G}(\mathrm{IgG})$ and Alexa 594 goat antimouse IgG (Molecular Probes) diluted 1/500 in blocking solution without NGS. Finally, coverslips were washed with PBS/0.1\% Triton X-100 and mounted with Mowiol 4-88 on glass slides. Preparations were examined using a Bio-Rad Microradiance confocal laser connected to a Zeiss Axioskop2 plus fluorescence microscope or using a Zeiss Axioskop2 plus fluorescence microscope, at a $600 \times$ magnification. Images were processed using Adobe Photoshop software.

\subsection{Nucleotide sequence accession number}

The sequence containing p10 gene from ASFV Lisbon 60 strain was deposited in GenBank under the accession number DQ275352.

\section{Results}

\subsection{ASFV p10 protein is imported into the nucleus of yeast cells}

To assess the nuclear import ability of ASFV p10 protein, a yeast-based nuclear import assay was used (Rhee et al., 2000). The underlying principle of this assay is the expression in yeast cells of a triple fusion protein encoded by pNIA, comprising mLexA (bacterial lexA modified to inactivate its internal NLS), Gal4AD (yeast Gal4p activation domain), and the protein to be tested, subcloned in-frame downstream of Gal4AD. The expression of the two reporter 
genes HIS3 and lacZ present in the L40 S. cerevisiae strain under the control of LexA (Hollenberg et al., 1995) depends on the intracellular localization of the tested fusion protein. If the tested protein contains a functional NLS, the fusion product is localized in the yeast cell nucleus. Yeast cells harboring this construct possess $\beta$-galactosidase activity and are able to grow in the absence of histidine as a consequence of lac $Z$ and HIS3 gene expression, respectively. Conversely, if the protein lacks a functional NLS, the fusion product is retained in the yeast cytoplasm, at least partly abolishing the B-galactosidase activity and impeding growth on histidine-deficient medium. The green fluorescent protein (GFP) encoding DNA was cloned upstream of p10 protein to ensure that the molecular weight of the fusion protein would be higher than $60 \mathrm{kDa}$, which is the diffusion limit of the nuclear pore complex (Görlich and Kutay, 1999), therefore excluding the possibility of any nucleus-cytoplasm diffusion.

As shown in Fig. 1a, yeast cells expressing the fusion product containing the ASFV p10 protein (pNIAGFPp10) were able to grow on histidine and tryptophan deficient medium, indicating that expression of the reporter HIS3 gene was induced. In addition, this construct induced B-galactosidase activity (Fig. 1a). pNIA + E2 was used as a positive control for the nuclear import assay. This plasmid encodes the protein VirE2, an Agrobacterium protein shown to lack a NLS functional in animal cells (Guralnick et al., 1996), fused to the NLS of SV40 large $\mathrm{T}$ antigen which is actively imported into the nucleus, thus leading to maximal expression of the two reporter genes. No yeast growth was observed for pNIAE2 and pNIAD2 antisense (pNIAD2(ant)), which were used as negative controls. pNIAE2 expresses VirE2, and pNIAD2 (ant) contains the cDNA sequence of VirD2, an Agrobacterium protein that carries a functional NLS (Citovsky et al., 1994), subcloned in the antisense orientation (Rhee et al., 2000).

To validate the qualitative results of $\beta$-galactosidase activity obtained by the filter lift assay, the liquid culture assay was used to quantify the activity of this enzyme in yeasts expressing the different constructs. Yeast cells harboring pNIAGFPp10 showed significantly higher levels of $\beta$-galactosidase activity than those observed for the negative controls (pNIAE2 and
pNIAD2(ant)) (Fig. 1b). pNIAGFP which encodes the fusion protein mLexA-Gal4AD-GFP, was used as a negative control. As expected, the presence of GFP did not have any effect on the levels of $\beta$-galactosidase activity (Fig. 1b).

Together, these results strongly suggest that p10 protein contains a functional NLS.

\subsection{ASFV p10 protein is imported into the nucleus of mammalian cells}

To confirm the results obtained using the yeastbased nuclear import assay in an ASFV native system, we performed studies on the subcellular localization of p10 protein in ASFV host mammalian cells (Vero cells). For this purpose, we generated a plasmid coding for a fusion protein that contains GFP and Bglucuronidase (GFPßGUS), thus being larger ( $\sim 94 \mathrm{kDa})$ than the size limit for diffusion through the NPC (Görlich and Kutay, 1999), and p10 protein was then subcloned at the C-terminus of BGUS. Fusion proteins were expressed in Vero cells by transient transfection, and protein intracellular localization was visualized by fluorescence microscopy.

As shown in Fig. 2, localization of the expressed GFPßGUS protein was exclusively cytoplasmic, in contrast to what was observed with GFP alone, which is distributed throughout the cell as a consequence of its molecular weight that allows its passive diffusion within the cell. However, it is interesting to observe that the fusion protein containing GFP, BGUS and p10 protein (GFPßGUSp10) accumulated strongly in cell nuclei (Fig. 2), providing clear evidence that ASFV p10 protein contains at least one NLS that is capable of mediating active protein transport from the cytoplasm to the nucleus in mammalian cells.

\subsection{Subcellular localization of different fragments of the ASFV p10 protein in mammalian cells}

To identify the localization of the functional nuclear import signal(s) within ASFV p10 protein, the subcellular localization of several GFP-tagged p10 fragments was analyzed in Vero cells by fluorescence microscopy (Fig. 3).

A detailed analysis of the amino acid sequence of ASFV p10 protein of the Lisbon 60 strain (GenBank 
$\beta$-Galactosidase activity (a)

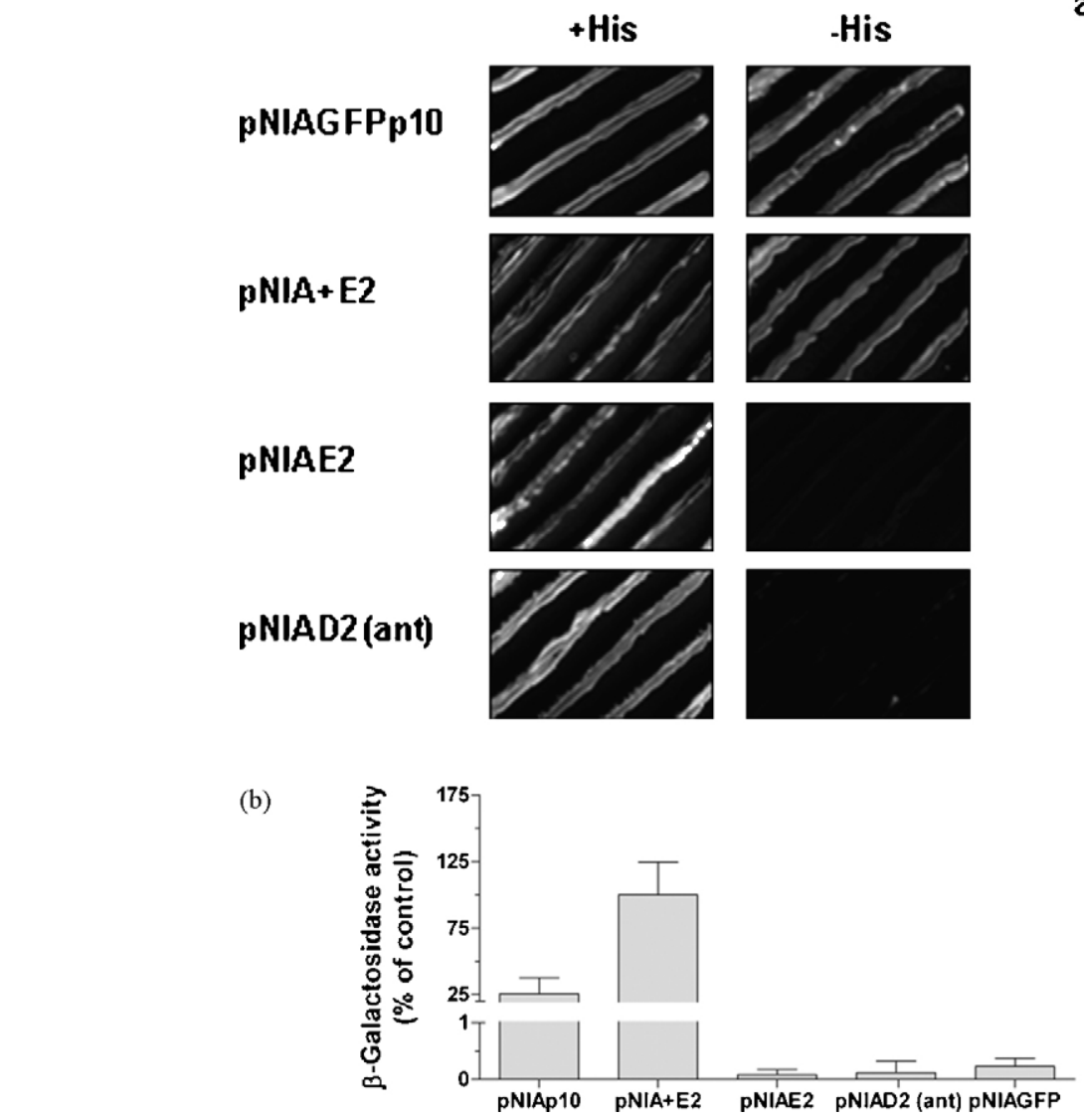

Yeast growth

Fig. 1. Evaluation of the nuclear import ability of ASFV p10 protein in yeast cells. (a) Evaluation of yeast cell growth and B-galactosidase activity. The growth of L40 yeasts transformed with the indicated constructs was analyzed both on selective medium without tryptophan (+His) and on medium without tryptophan and histidine (-His). The ß-galactosidase activity was evaluated using the colony-lift filter assay. (b) Quantification of $ß$-galactosidase activity in liquid cultures. The data are expressed as a percentage of maximal enzymatic activity obtained for pNIA + E2. Standard deviations are shown based on triplicates of at least three independent experiments.

accession number DQ275352) led to the identification of a lysine and arginine rich sequence between amino acids 71 and 77 (KKIKRSK), this constituting, therefore, a potential candidate to mediate the nuclear import of $\mathrm{p} 10$ protein. This sequence is also present in ASFV p10 protein of BA71V strain (Muñoz et al., 1993) (Fig. 4), and resembles the most commonly known classical NLSs which are composed of 4-6 K/ $\mathrm{R}$ amino acids, similar to the NLS of SV40 large T antigen (Kalderón et al., 1984). In contrast to what was observed for the full-length protein (GFPßGUSp10), the fragment GFPßGUS (1-70) lost the exclusivity of nuclear localization (compare Figs. 2 and 3), indicating that although not alone, the sequence between amino acids 71 and 77 may be involved in the active nuclear import of $\mathrm{p} 10$ protein, as predicted by the amino acid sequence analysis. Unexpectedly, the fragments GFPßGUS (31-77) and GFPßGUS (54-77) were not imported into the nucleus of transfected cells (Fig. 3), clearly indicating that the presence of the sequence between amino acids 71 and 77 is not sufficient to mediate the active nuclear import of $\mathrm{p} 10$ protein.

Analysis of the nuclear import ability of several other p10 fragments (GFPßGUS(1-30), GFPßGUS(1-16), GFPßGUS(9-70), GFPßGUS (31-70), GFPßGUS(30-53), GFPßGUS(30-40) and GFPßGUS(40-53)) (Fig. 3), did not allow the 


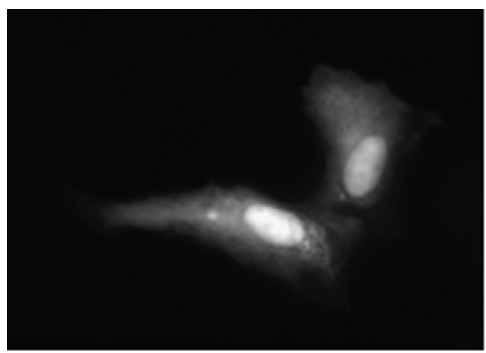

GFP

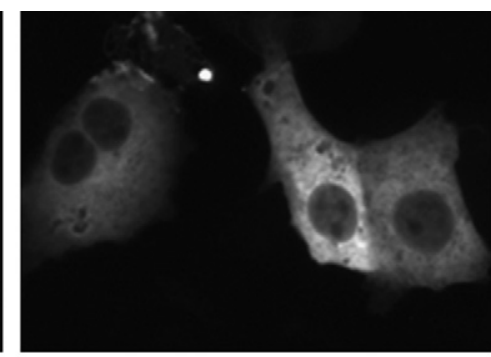

GFPßGUS

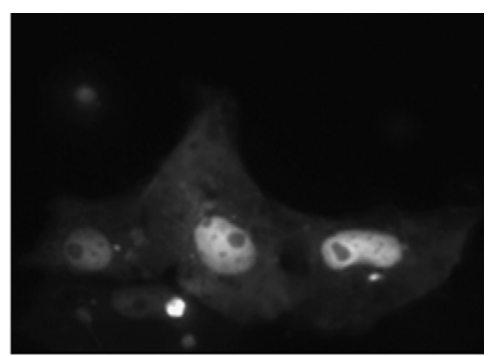

GFPßGUSp10

Fig. 2. Evaluation of the nuclear import ability of ASFV p10 protein in mammalian cells. Subconfluent cultures of Vero cells were transiently transfected with the plasmids encoding GFP, GFPßGUS or GFPßGUSp10. Thirty-six hours after transfection, the cells were fixed and the subcellular localization of the different proteins was analyzed by fluorescence microscopy (magnification $600 \times$ ). Experiments were performed at least two times and representative images are shown.

identification of any other stretch of amino acids responsible for the nuclear import of p10 protein, since all these fragments exhibited an essentially cytoplasmic localization. On the other hand, the p10 protein fragments that contain the N-terminal of the protein (GFPßGUS(1-51), GFPßGUS(1-62) and GFPßGUS(1-70)) exhibited partial nuclear localization (Fig. 3). Curiously, the fragment GFPßGUS(977) was the only one that exhibited a nuclear localization similar to that observed for the fulllength p10 protein (Fig. 3).

Taken together, these results strongly suggest that the functional NLS of ASFV p10 protein is not simply composed of a linear (either continuous or bipartite) stretch of amino acids. Instead, it seems to be dependent on a specific and complex three-dimensional protein folding pattern.

\subsection{ASFV p10 protein accumulates in the cell nucleus during viral infection}

To investigate whether p10 protein is actively imported into the nucleus of ASFV-infected cells, immunofluorescence experiments were performed in virus-infected Vero cells, which were fixed at different times post-infection. As a control we also monitored the intracellular distribution of ASFV p72, which is the major ASFV capsid protein and whose expression occurs during the late phase of the infection cycle (Cistué and Tabarés, 1992).

Fig. 5 shows the images obtained by confocal fluorescence microscopy from experiments involving a double labeling with the rabbit polyclonal serum against p10 protein (upper panels) and the mouse monoclonal antibody against protein p72 (lower panels). During the first $8 \mathrm{~h}$ post-infection (hpi), p10 protein although poorly detected, was confined to the incoming virus particles, similarly to what was observed for $\mathrm{p} 72$ protein. On the other hand, at $10 \mathrm{hpi}$ and at later times p10 protein was localized almost exclusively in the nucleus of the infected cells, although some labeling was also visualized in the viral factories. Anti-p72 antibodies strongly labeled the virus factories, as well as virus particles scattered throughout the cytoplasm at late times post-infection, as observed previously (Andrés et al., 2001). Since p10 is a structural protein, it would be expected to detect it more strongly at the viral factories where viral assembly takes place, as observed for protein $\mathrm{p} 72$.

It should be emphasized that in the present study we fixed the cells with paraformaldehyde and methanol. Methanol fixation has been reported to cause precipitation of cellular proteins and thus should overcome the problem of protein redistribution due to insufficient crosslinking. Moreover, methanol fixation washes out soluble cellular proteins (Brock et al., 1999), disrupts the cytoskeleton and leads to extraction of antigen and membrane lipids (Vekemans et al., 2004). On the other hand, paraformaldehyde provides good stabilization of permeabilized membranes, preventing the linkage of intracellular proteins with extracellular molecules and increases the indirect fluorescence of intranuclear antigens (Pollice et al., 1992). To combine the advantages of both procedures, methanol fixation preceded by paraformaldehyde crosslinking, as performed in our study, has been 
(a)

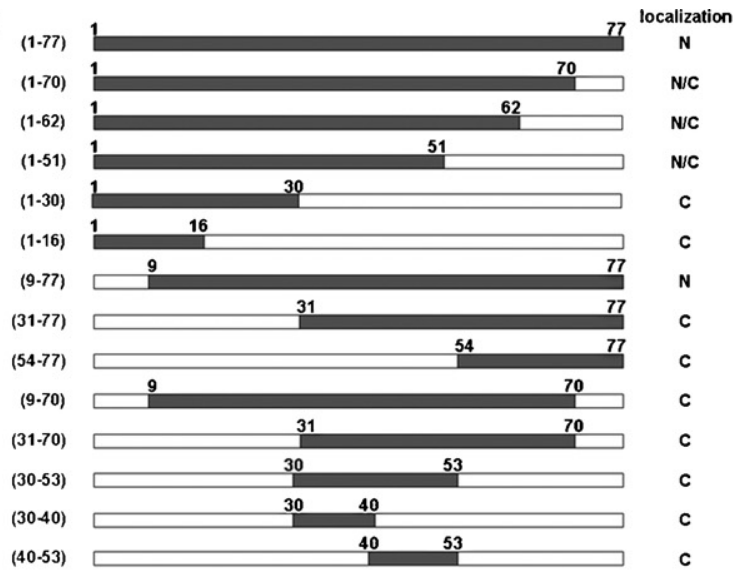

(b) GFP $\beta$ GUS (1-70)

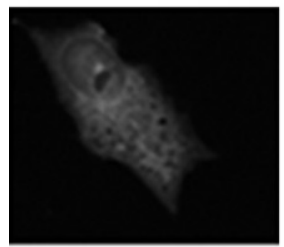

GFPßGUS

(9-77)

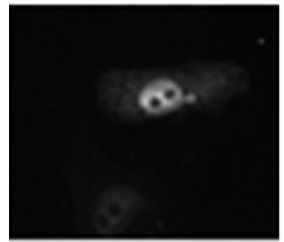

GFPßGUS

(30-53)

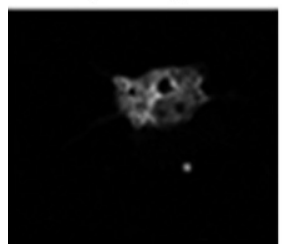

GFP $\beta$ GUS

(1-62)

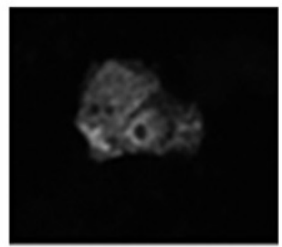

GFPßGUS

(31-77)

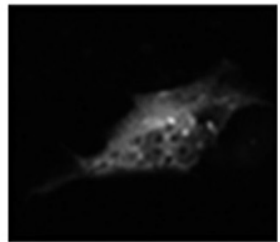

GFP $\beta$ GUS

(30-40)

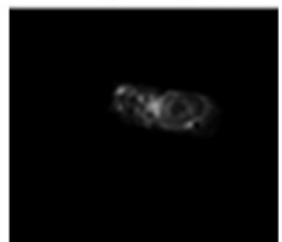

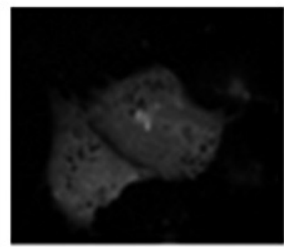

GFPßGUS

(54-77)

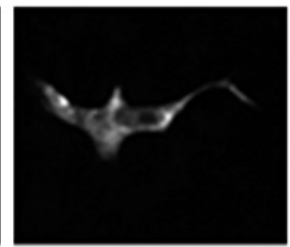

GFP $\beta$ GUS

(40-53)

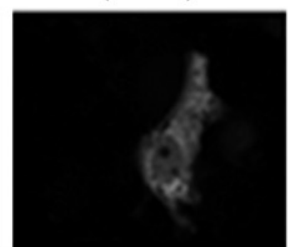

GFPBGUS

(1-30)

GFPBGUS

(1-16)

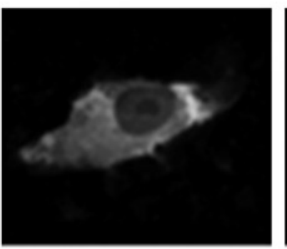

GFPBGUS

(9-70)
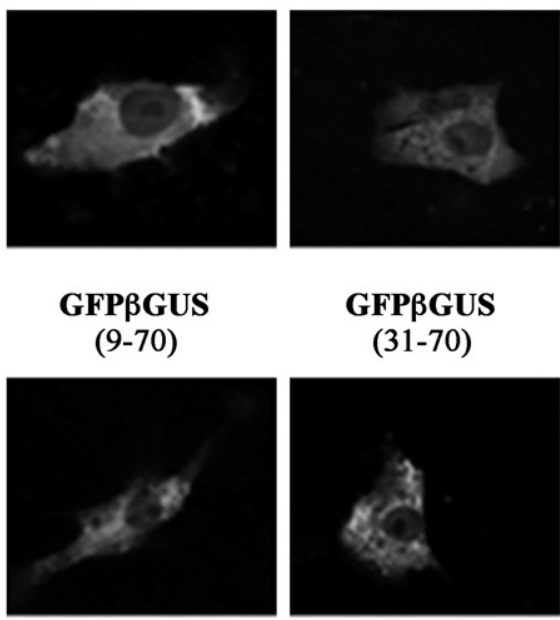

GFPßGUS

(31-70)

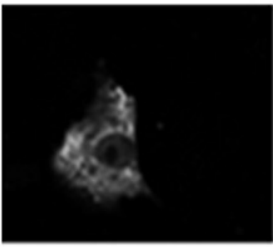

Fig. 3. Subcellular localization of different fragments of the ASFV p10 protein. (a) Schematic diagram of the different fragments of ASFV p10 protein and their subcellular localization in Vero cells: $\mathrm{C}, \mathrm{C} / \mathrm{N}$ and $\mathrm{N}$ indicate that, in the majority of the transfected cells, the fusion protein being tested presents an exclusively cytoplasmic, both cytoplasmic and nuclear, or nuclear localization, respectively. (b) Subcellular distribution of the different fragments of p10 protein evaluated by fluorescence microscopy (magnification $600 \times$ ). Vero cells were fixed $36 \mathrm{~h}$ following transfection with the indicated constructs. Experiments were performed at least two times and representative images are shown. 


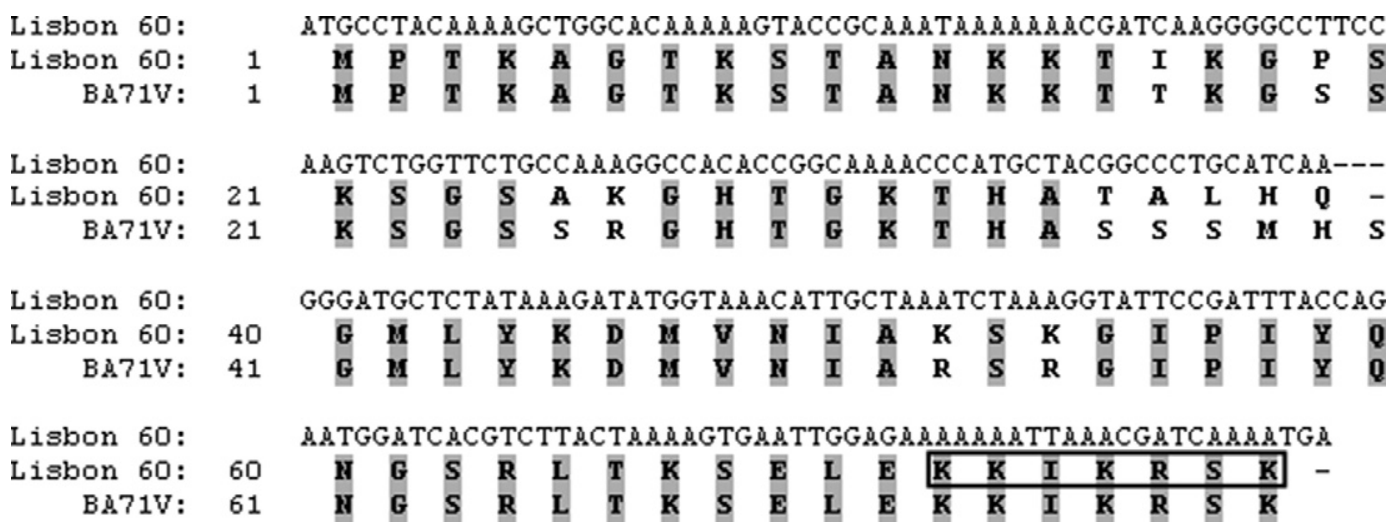

Fig. 4. Nucleotide sequence that codes for ASFV p10 protein in Lisbon 60 strain, and its respective amino acid sequence. The amino acid sequence of ASFV p10 protein of BA71V strain is represented for comparison. Common amino acids to both strains are highlighted in grey. Amino acids comprised between positions 71 and 77 of ASFV p10 protein in Lisbon 60 strain, are inserted in a rectangle.

described as a good option for the fixation of transmembrane and soluble cytoplasmic proteins (Pollice et al., 1992; Brock et al., 1999; Hoetelmans et al., 2001). Nevertheless, to investigate whether the low detection of p10 protein in the viral factories was due to the use of methanol, we determined p10 localization in the ASFV-infected cells fixed both solely with paraformaldehyde and with paraformaldehyde and methanol, using fluorescence microscopy. As observed in Fig. 6, no significant differences were detected using both procedures. However, the presence of p10 in the viral factories was more evident in the images obtained by fluorescence microscopy than in those by confocal fluorescence microscopy (compare Figs. 5 and 6), confirming the presence of this structural protein in the viral assembly sites, as it would be expected.

\section{Discussion}

The gene encoding p10 protein of ASFV strain BA71V was first described by Muñoz et al. (1993). This small protein $(\sim 10 \mathrm{kDa})$ is a structural protein that exhibits a strong DNA binding activity with similar affinity for both double- and single-stranded DNA. Moreover, it contains a large number of basic amino acids (Muñoz et al., 1993), is localized at the virus nucleoid (Andrés et al., 2002) and might be involved in DNA packaging. In face of these characteristics, we hypothesized that this viral protein may assist in the transport of ASFV DNA into the host cell nucleus during the viral infection cycle.

The active nuclear import ability of $\mathrm{p} 10$ protein was assessed by using the described yeast-based nuclear import assay (Rhee et al., 2000). Using this approach we were able to demonstrate that p10 protein is actively imported into the nucleus of yeast cells, as illustrated by the results showing that 10 protein is capable of directing nuclear accumulation of a large fusion protein, comprising mLexA, Gal4AD and GFP.

These results were validated in a more relevant biological system by determining the intracellular localization of p10 protein in mammalian cells by fluorescence microscopy. It should be noted that the fusion protein containing $\mathrm{p} 10$ protein (GFP $\beta$ GUSp10) has a molecular weight above the limit for diffusion ( $\sim 60 \mathrm{kDa}$ ) through the nuclear pore complex (Görlich and Kutay, 1999). In agreement with the results obtained in yeasts, Vero cells transfected with GFPßGUSp10 exhibited fluorescence almost exclusively inside the cell nucleus. It is interesting to note that this observation is in contrast to what was obtained in cells transfected with GFP $\beta$ GUS, where the fluorescence is detected exclusively in the cell cytoplasm. These results show that, even being smaller than the diffusion limit of the NPC, ASFV p10 protein is able to exploit cellular mechanisms for active nuclear import.

Interestingly, the highly basic region comprised between amino acids 71 and 77 of p10 protein (KKIKRSK), which resembles other described NLSs 


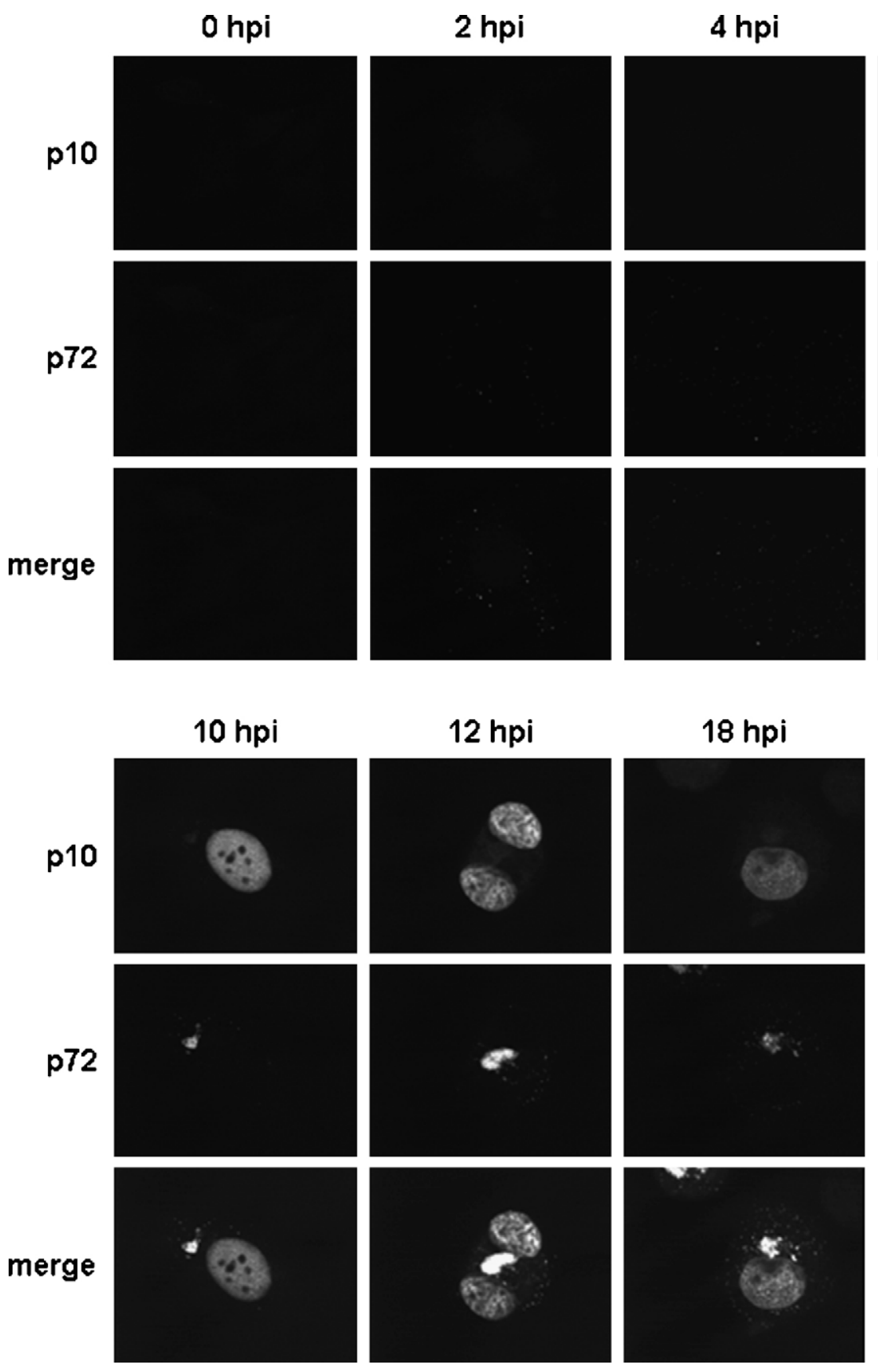

Fig. 5. Immunofluorescence microscopy of ASFV-infected cells. Vero cells infected with ASFV BA71V strain were fixed with $2 \%$ paraformaldehyde for $1 \mathrm{~h}$ at room temperature and then with methanol at $-20{ }^{\circ} \mathrm{C}$ for $5 \mathrm{~min}$ and processed for immunofluorescence analysis at the indicated times post-infection. Samples were double-labeled with rabbit serum anti-p10 and mouse monoclonal antibody 17LD3 anti-p72, followed by incubation with secondary antibodies conjugated to Alexa 488 and 594, respectively. Confocal laser scanning images acquired are presented both separately and merged. Experiments were performed at least two times and representative images are shown. 


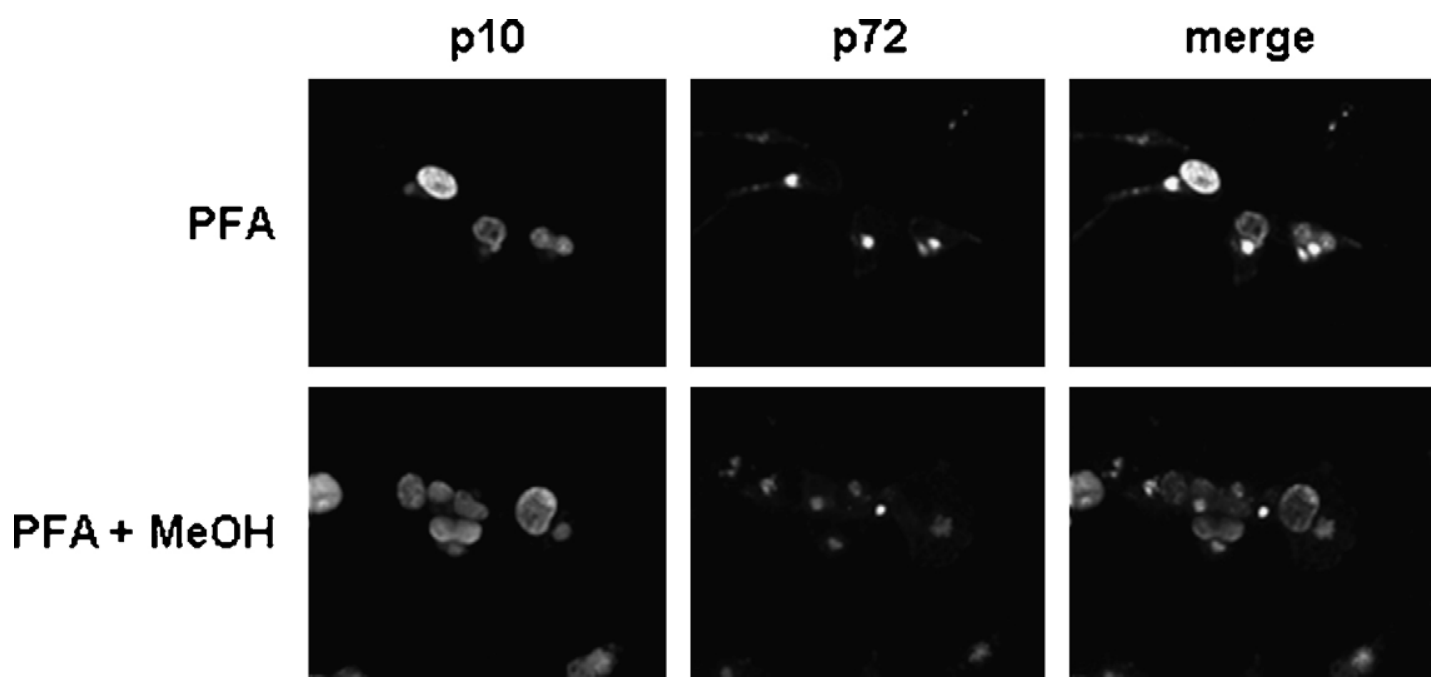

Fig. 6. p10 protein localization in ASFV-infected cells using different fixation conditions. Vero cells infected with ASFV BA71V strain were fixed with $2 \%$ paraformaldehyde for $1 \mathrm{~h}$ at room temperature (PFA) or with $2 \%$ paraformaldehyde for $1 \mathrm{~h}$ at room temperature and then with methanol at $-20{ }^{\circ} \mathrm{C}$ for $5 \mathrm{~min}(\mathrm{PFA}+\mathrm{MeOH})$. In both cases, the cells were processed for immunofluorescence analysis at $18 \mathrm{hpi}$. Samples were double-labeled with rabbit serum anti-p10 and mouse monoclonal antibody 17LD3 anti-p72, followed by incubation with secondary antibodies conjugated to Alexa 488 and 594, respectively. Fluorescence microscopy images (magnification $600 \times$ ) acquired are presented both separately and merged. Experiments were performed at least two times and representative images are shown.

like the classical NLS of SV40 large T antigen (PKKKRKV) (Kalderón et al., 1984) and the yeast histone 2B (GKKRSKA) (Moreland et al., 1987), was required for exclusive nuclear localization, as inferred from the observation that the fragment GFPßGUS $(1-$ 70) lost the exclusivity of nuclear localization. However, the presence of this sequence was not sufficient to induce protein nuclear import since the constructs GFP $\beta$ GUS(31-77) and GFP $\beta G U S(54-77)$ exhibited exclusively cytoplasmic fluorescence. The constructs GFP $\beta G U S(1-70)$, GFP $\beta$ GUS(1-62) and GFP $\beta$ GUS(1-51) that do not contain the stretch of amino acids 71-77 exhibited fluorescence both in the cytoplasm and in the nucleus, indicating that although not sufficient to mediate an exclusive nuclear localization, the first 51 amino acids of ASFV p10 protein are also involved in the active protein nuclear import. However, since the constructs GFP $\beta G U S(1-$ 16) and GFPßGUS(1-30) exhibited exclusively cytoplasmic fluorescence, it was expected that amino acids comprised between positions 31 and 51 could promote at least partial protein nuclear localization. Nevertheless, this hypothesis was not confirmed since the constructs GFP $\beta$ GUS $(30-40)$, GFP $\beta$ GUS(40-53), GFP $\beta G U S(30-53)$, GFP $\beta G U S(31-70)$ and GFPßGUS(9-70) exhibited exclusively cytoplasmic fluorescence. We observed that solely the constructs GFP $\beta$ GUSp 10 and GFP $\beta$ GUS(9-77), both containing the stretch of amino acids from position 71 to 77 , exhibited exclusive nuclear localization.

To summarize, the results of the present work provide evidence that the amino acids comprised between positions 71 and 77 are important, although not sufficient, for the active nuclear import of ASFV p10 protein. It is possible that the role of this sequence depends also on the presence of discontinuous epitopes that may come together to form a specific three-dimensional arrangement of amino acids on the protein surface upon its folding, as observed for other proteins (Baake et al., 2001). It should be noted that the three-dimensional structure of a protein might be severely affected when the protein is expressed as a fusion protein, as is the case in our study. Further studies, namely the determination of the threedimensional structure, may help to identify putative amino acids responsible for the active nuclear import of ASFV p10 protein.

The results from immunofluorescence studies in ASFV-infected Vero cells (strain BA71V) demonstrate that $\mathrm{p} 10$ protein is localized inside the nucleus at 
$10 \mathrm{hpi}$ and also at later times (12 and $18 \mathrm{hpi}$ ). These results validate those obtained from studies involving transfection of the cells with p10 protein, confirming that this ASFV protein contains a functional NLS. In this context, it is relevant to point out that the amino acid sequences of ASFV p10 protein from the BA71V (GenPept accession number S29971) and Lisbon 60 strains, deposited in GenBank under the accession number DQ275352, Exhibit 85\% amino acid identity, and that the sequence containing the last seven amino acids, identified as a potential NLS, display $100 \%$ identity in both strains. To our knowledge, the sequence of ASFV p10 protein of other strains has not yet been described.

It has been described that ASFV viral DNA synthesis is initiated inside the nucleus, a stage that is followed by a longer replication phase that occurs in the cytoplasm. The nuclear phase takes place during the early times post-infection, namely, during the first 8 h (García-Beato et al., 1992; Rojo et al., 1999). The fact that we were unable to detect p10 inside the nucleus of infected cells, by immunofluorescence, during the first $8 \mathrm{~h}$ post-infection does not support our hypothesis that ASFV p10 protein would be involved in the active transport of viral DNA into the host cell nucleus during the viral infection cycle. Further studies need to be addressed in order to understand the role of p10 protein during the replication cycle of ASFV. However, the observation that p10 strongly accumulates inside the nucleus of infected cells and that this protein has a functional NLS, which may induce a more rapid and efficient nuclear localization of the protein than that achieved via simple diffusion, raises the interesting possibility that ASFV p10 protein may play a role inside the nuclear compartment during the late phase of the viral infection cycle.

\section{Acknowledgments}

This work was supported by a grant from the Portuguese Foundation for Science and Technology (POCTI/CVT/44854/2002). I. Nunes-Correia and A. Eulálio were recipients of fellowships from the Portuguese Foundation for Science and Technology. The work in V. Citovsky laboratory was supported by grants from the National Institutes of Health, National
Science Foundation, US Department of Agriculture, US-Israel Binational Science Foundation (BSF), and US-Israel Binational Research and Development Fund (BARD). The work in M.L. Salas laboratory was supported by a grant from the Spanish Ministerio de Educatión y Ciencia (BFU2004-00298) and by an institutional grant from the Fundación Ramón Areces. J.M. Rodríguez was supported by the "Ramón y Cajal" program of the Ministerio de Educación $y$ Ciencia.

\section{References}

Andrés, G., Símon-Mateo, C., Viñuela, E., 1997. Assembly of African swine fever virus: role of polyprotein pp220. J. Virol. 71, 2332-2341.

Andrés, G., García-Escudero, R., Simón-Mateo, C., Viñuela, E., 1998. African swine fever virus is enveloped by a two-membraned collapsed cisterna derived from the endoplasmic reticulum. J. Virol. 72, 8988-9001.

Andrés, G., García-Escudero, R., Viñuela, E., Salas, M.L., Rodriguez, J.M., 2001. African swine fever virus structural protein $\mathrm{pE} 120 \mathrm{R}$ is essential for virus transport from assembly sites to plasma membrane but not for infectivity. J. Virol. 75, 67586768.

Andrés, G., García-Escudero, R., Salas, M.L., Rodriguez, J.M., 2002. Repression of African swine fever virus polyprotein pp220-encoding gene leads to the assembly of icosahedral core-less particles. J. Virol. 76, 2654-2666.

Baake, M., Doenecke, D., Albig, W., 2001. Characterisation of nuclear localisation signals of the four human core histones. J. Cell. Biochem. 81, 333-346.

Breeden, L., Nasmyth, K., 1985. Regulation of the yeast HO gene. Cold Spring Harb. Symp. Quant. Biol. 50, 643-650.

Breese Jr., S.S., Pan, I.C., 1978. Electron microscopic observation of African swine fever virus development in Vero cells. J. Gen. Virol. 40, 499-502.

Brock, R., Hamelers, I.H.L., Jovin, T.M., 1999. Comparison of fixation protocols for adherent cultured cells applied to a GFP fusion protein of the epidermal growth factor receptor. Cytometry $35,353-362$.

Carrascosa, A.L., del Val, M., Santarén, J.F., Viñuela, E., 1985. Purification and properties of African swine fever virus. J. Virol. 54, 337-344.

Carrascosa, J.L., Carazo, J.M., Carrascosa, A.L., García, N., Santisteban, A., Viñuela, E., 1984. General morphology and capsid fine structure of African swine fever virus particles. Virology 132, 160-172.

Cistué, C., Tabarés, E., 1992. Expression in vivo and in vitro of the major structural protein (VP73) of African swine fever virus. Arch. Virol. 123, 111-124.

Citovsky, V., Warnick, D., Zambrysky, P., 1994. Nuclear import of Agrobacterium VirD2 and VirE2 proteins in maize and tobacco. Proc. Natl. Acad. Sci. U.S.A. 91, 3210-3214. 
Dingwall, C., Robbins, J., Dilworth, S.M., Roberts, B., Richardson, W.D., 1988. The nucleoplasmin nuclear location sequence is larger and more complex than that of SV-40 large T antigen. J. Cell. Biol. 107, 841-849.

Dingwall, C., Laskey, R.A., 1991. Nuclear targeting sequences-a consensus? Trends Biochem. Sci. 16, 478-481.

Dixon, L.K., Abrams, C.C., Bowick, G., Goatley, L.C., Kay-Jackson, P.C., Chapman, D., Liberan, E., Nix, R., Silo, R., Zhang, F., 2004. African swine fever virus proteins involved in evading host defence systems. Vet. Immunol. Immunop. 100, 117-134.

Eulálio, A., Nunes-Correia, I., Carvalho, A.L., Faro, C., Citovsky, V., Simões, S., Pedroso de Lima, M.C., 2004. Two African swine fever virus proteins derived from a common precursor exhibit different nucleo-cytoplasmic transport activities. J. Virol. 78, 9731-9739.

García-Beato, R., Salas, M.L., Viñuela, E., Salas, J., 1992. Role of the host cell nucleus in the replication of African swine fever virus DNA. Virology 188, 637-649.

Görlich, D., Kraft, R., Kostka, S., Vogel, F., Hartmann, E., Laskey, R.A., Mattaj, I.W., Izaurraide, E., 1996. Importin provides a link between nuclear protein import and U snRNA export. Cell 87, 21-32.

Görlich, D., Kutay, U., 1999. Transport between the cell nucleus and the cytoplasm. Annu. Rev. Cell Dev. Biol. 15, 607-660.

Guralnick, B., Thomsen, G., Citovsky, V., 1996. Transport of DNA into the nuclei of Xenopus oocytes by a modified VirE2 protein of Agrobacterium. Plant Cell 8, 363-373.

Hoetelmans, R.W., Prins, F.A., Cornelese-ten Velde, I., van der Meer, J., van de Velde, C.J., van Dierendonck, J.H., 2001. Effects of acetone, methanol, or paraformaldehyde on cellular structure, visualized by reflection contrast microscopy and transmission and scanning electron microscopy. Appl. Immunohistochem. Mol. Morphol. 9, 346-351.

Hollenberg, S.M., Sternglanz, R., Cheng, P.F., Weintraub, H., 1995. Identification of a new family of tissue-specific basic helix-loophelix proteins with a two-hybrid system. Mol. Cell. Biol. 15, 3813-3822.

Kaiser, C., Michaelis, S., Mitchell, A., 1994. Methods in Yeast Genetics. Cold Spring Harbor Laboratory Press, Cold Spring Harbor, NY.

Kalderón, D., Richardson, W.D., Markham, A.F., Smith, A.E., 1984. Sequence requirements for nuclear location of simian virus 40 large-T antigen. Nature 311, 33-38.

Leitão, A., Cartaxeiro, C., Coelho, R., Cruz, B., Parkhouse, R.M.E., Portugal, F.C., Vigário, J.D., Martins, C.L.V., 2001. The nonhaemadsorbing African swine fever virus isolate ASFV/NH/P68 provides a model for defining the protective anti-virus immune response. J. Gen. Virol. 82, 513-523.

Lyons, R.H., Ferguson, B.Q., Rosenberg, M., 1987. Pentapeptide nuclear localization signal in adenovirus E1a. Mol. Cell. Biol. 7, 2451-2456.
Macara, I.G., 2001. Transport into and out of the nucleus. Microbiol. Mol. Biol. Rev. 65, 570-594.

Makkerh, J.P., Dingwall, C., Laskey, R.A., 1996. Comparative mutagenesis of nuclear localization signals reveals the importance of neutral and acidic amino acids. Curr. Biol. 6, 1025-1027.

Michael, W.M., Eder, P.S., Dreyfuss, G., 1997. The K nuclear shuttling doma: a novel signal for nuclear import nuclear export in the hnRNP K protein. EMBO J. 16, 3587-3598.

Miller, J.H, 1972. Experiments in Molecular Genetics. Cold Spring Harbor Laboratory Press, Cold Spring Harbor, NY.

Moreland, R.B., Langevin, G.L., Singer, R.H., Garcea, R.L., Hereford, L.M., 1987. Amino acid sequences that determine the nuclear localization of yeast histone 2B. Mol. Cell. Biol. 7, 4048-4057.

Muñoz, M., Freije, J.M., Salas, M.L., Viñuela, E., López-Otin, C., 1993. Structure and expression in E. coli of the gene coding for protein p10 of African swine fever virus. Arch. Virol. 130, 93-107.

Ortin, J., Viñuela, E., 1977. Requirement of cell nucleus for African swine fever virus replication in Vero cells. J. Virol. 21, 902-905.

Pollice, A., McCoy, J., Shackney, S., Smith, C., Agarwal, J., Burholt, D., Janocko, L., Hornicek, F., Singh, S., Hartsock, R., 1992. Sequential paraformaldehyde and methanol fixation for simultaneous flow cytometric analysis of DNA, cell surface proteins, and intracellular proteins. Cytometry 13, 432-444.

Rhee, Y., Gurel, F., Gafni, Y., Dingwall, C., Citovsky, V., 2000. A genetic system for detection of protein nuclear import and export. Nat. Biotechnol. 18, 433-437.

Rojo, G., Garcia-Beato, R., Viñuela, E., Salas, M.L., Salas, J., 1999. Replication of African swine fever virus DNA in infected cells. Virology 257, 524-536.

Salas, J., Salas, M.L., Viñuela, E., 1999. African swine fever virus: a missing link between poxviruses and iridoviruses. In: Domingo, E., Webster, R., Holland, J. (Eds.), Origin and Evolution of Viruses. Academic Press, Inc., New York, pp. 467-480.

Sanz, A., García-Barreno, B., Nogal, M.L., Viñuela, E., Enjuanes, L., 1985. Monoclonal antibodies specific for African swine fever virus proteins. J. Virol. 54, 199-206.

Stachel, S.E., An, G., Flores, C., Nester, E.W., 1985. A Tn3 lacZ transposon for the random generation of $\beta$-galactosidase gene fusions: application to the analysis of gene expression in Agrobacterium. EMBO J. 4, 891-898.

Vekemans, K., Rosseel, L., Wisse, E., Braet, F., 2004. Immunolocalization of Fas and FasL in rat hepatic endothelial cells: influence of different fixation protocols. Micron 35, 303-306.

Whittaker, G.R., Helenius, A., 1998. Nuclear import and export of viruses and virus genomes. Virology 246, 1-23.

Yáñez, R.J., Rodriguez, J.M., Nogal, M.L., Yuste, L., Enriquez, C., Rodríguez, J.F., Viñuela, E., 1995. Analysis of the complete nucleotide sequence of African swine fever virus. Virology 208, 249-278. 